\title{
Surgical Management of Chronic and Refractory TMJ Dislocation in Medically Compromised Patients
}

\author{
Reda Elgazzar ${ }^{1^{*}}$ and Christopher Ward ${ }^{2}$ \\ ${ }^{1}$ Oral and Maxillofacial Surgery, Dental Diagnostic \& Surgical Sciences, Bannatyne Ave, Winnipeg, Manitoba, Canada \\ ${ }^{2}$ Oral and Maxillofacial Surgery, Toronto University, Toronto, Canada
}

*Corresponding author: Reda Elgazzar, Oral and Maxillofacial Surgery, Dental Diagnostic \& Surgical Sciences, Bannatyne Ave, Winnipeg, Manitoba, Canada, Tel: +1(204)789-3634; Fax: +1(204)272-3077; E-mail: reda.elgazzar@umanitoba.ca

Received date: August 09, 2018; Accepted date: September 07, 2018; Published date: September 10, 2018

Copyright: ( 2018 Elgazzar R, et al. This is an open-access article distributed under the terms of the Creative Commons Attribution License, which permits unrestricted use, distribution, and reproduction in any medium, provided the original author and source are credited.

\begin{abstract}
Recurrent and long-standing chronic dislocations of the Temporomandibular Joint (TMJ) are relatively rare conditions that have a profoundly negative impact on a patient's quality of life. To date, no definitive protocols or guidelines exist regarding the appropriate management of these conditions. Many diverse methods have been employed ranging from non-surgical conservative approaches to invasive surgical procedures to correct recurrent and chronic long-standing dislocations. In this article, two different cases are presented; case \#1 a chronic recurrent TMJ dislocation in association with Ehlers-Danlos syndrome; and case \#2, a chronic protracted TMJ dislocation in the setting of myotonic dystrophy. These two cases were successfully treated using two different treatment modalities after conservative management had failed. In case \#1, a combination of bilateral eminence augmentation utilizing L-shaped reconstruction plates, TMJ capsular augmentation with a temporalis myofascial flap as well as lateral pterygoid myotomy was employed. In case \#2, a condylectomy was used to resolve the long-standing dislocation. Both patients have not demonstrated any functional limitations or recurrence 2 years after surgical intervention. From these observations, we support utilizing these two techniques in the surgical management of chronic and refractory dislocation respectively.
\end{abstract}

Keywords: TMJ dislocation; TMJ Surgery; Ehlers-Danlos syndrome; Myotonic dystrophy

\section{Introduction}

Dislocation of the TMJ typically results in the complete loss of a normal articulating relationship between the condyle and the articular fossa of the temporal bone. Typically, the condyle is anteriorly displaced from the glenoid fossa, moving beyond the normal physiological range of motion, resulting in a new anterosuperior position relative to the articular eminence [1]. Causative factors for TMJ dislocation include: trauma, excessive mouth opening during yawning, laughing, vomiting, or lengthy dental procedures. Laxity in articular ligaments or the capsule, syndromes such as Marfan's syndrome and Ehlers-Danlos syndrome that cause connective tissue and joint laxity, as well as altered anatomical or dysfunctional components of the TMJ, have also been identified as factors of TMJ dislocation [2]. TMJ dislocation can be classified as acute, chronic recurrent or chronic protracted dislocation [3]. Typically, acute dislocations can be reduced in an outpatient clinic or hospital setting with little to no complications. However, in cases of chronic longstanding dislocations, physiological changes and remodelling events in the TMJ can lead to significant difficulty in providing treatment. The formation of fibrotic adhesions in the TMJ and associated structures as well as fibrous changes in the muscles of mastication and associated ligaments has been identified in long-standing dislocations. These fibrotic changes prevent the condyle from being reduced back into its physiologic position [4]. Numerous non-invasive and surgical methods to treat TMJ dislocations have been successfully employed and described to date. Invasive surgical procedures employed to treat chronic recurrent dislocations can be broadly classified into procedures that limit or enhance the condylar path, while surgical procedures used to treat chronic protracted dislocations aim to reduce the condyle by surgical repositioning, recontouring, or by its removal altogether [1]. Making an effective decision in regards to appropriate management of chronic TMJ dislocation is difficult due to the rarity of this condition and the number of surgical methods that have been successfully used in treatment. This article describes two clinical cases where chronic recurrent dislocation and a chronic protracted dislocation were successfully resolved by employing two different surgical procedures. To date, both patients continue to demonstrate excellent post-operative results with no reoccurrence of dislocations since their respective surgeries.

\section{Case 1}

An 18-year-old female with a history of chronic recurrent TMJ dislocation was referred to our services for proper management of her condition. The frequency of her TMJ dislocations was attributed to her underlying condition of Ehlers-Danlos Syndrome Type 3, causing articular hypermobility of the TMJs. Initial conservative approaches employing Intermaxillary Fixation (IMF) subsequent to reduction yielded only temporary success [5]. Due the frequency of her dislocations, the need for more definitive treatment became apparent. After obtaining consent, the patient was scheduled for the injection of autologous blood into both TMJs and respective capsules under sedation. IMF screws and elastics were placed bilaterally in the patient's maxilla and mandible following the injections to stabilize her occlusion and to seat the condyles within the glenoid fossa. Elastics were kept in place for four weeks after the procedure. After three months, the patient presented again to our services following another episode of dislocation. A decision was then made to perform a bilateral 
closed reduction of the TMJ with bilateral injection of a sclerosing agent, sodium tetradecyl sulfate, under general anesthesia. Arch bars were placed in the maxilla and mandible and secured using 24-guage wire. $2 \mathrm{~mL}$ of sodium tetradecyl sulfate was then injected into each joint capsule after manual reduction was accomplished. Four months after this treatment, the patient presented again after another episode of dislocation. As multiple conservative treatments failed to resolve the patient's chronic dislocations, the decision was made to employ a surgical approach. After some discussion, the patient consented to bilateral TMJ eminence reconstruction in combination with myofascial flap augmentation of her TMJ joint capsules as well as lateral pterygoid myotomy. Under general anesthesia, the left TMJ was exposed using an extended pre-auricular approach with an anterosuperior (hockeystick) extension. A nerve stimulator was used throughout the approach to ensure the facial nerve was not damaged. After the left condyle had been exposed, dissection of the lateral capsule off the condylar fossa and anterior eminence was performed. A myotomy of the lateral pterygoid was also carried out at this time. A $2.8 \mathrm{~mm}$ reconstruction plate curved into an L profile was then fixed to the anterior eminence using two $8 \mathrm{~mm}$ screws (Figure 1). After verifying good fixation of the plate, the position and condition of the articular disc was then inspected. The disc was noted to be in an acceptable anatomic position with no signs of perforation. The lateral capsule was then reattached to the eminence, and a temporalis myofascial rotational flap was then rotated inferiorly and fixed into position with a 3-0 vicryl suture to reinforce the lateral capsule. Good fixation and vascular supply of the flap was observed. Subcutaneous and a temporoparietal fascia were resuspended using 3-0 Vicryl buried stitches and the skin was closed using interrupted 4-0 Monocryl sutures. The patient was also placed in IMF using four $12 \mathrm{~mm}$ IMF screws and elastics. After 3 months, the entire procedure was repeated for her right TMJ. Overall, her postoperative course was uneventful, with no reoccurrence of dislocation in the 12 months since her surgery.

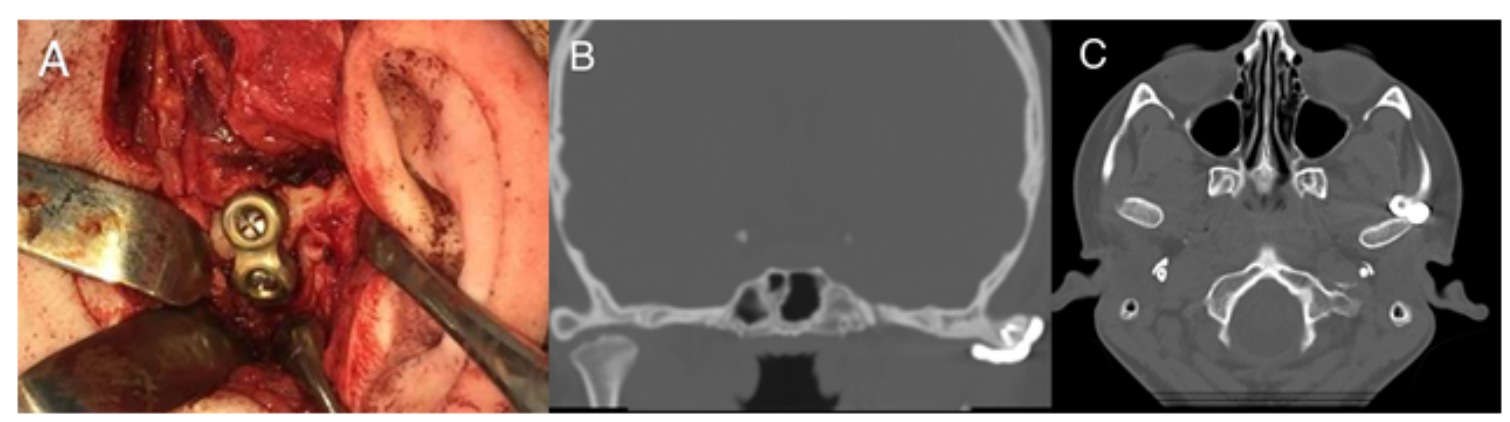

Figure 1: (A) Intraoperative photo; (B) Coronal and axial (C) postoperative CT scan views demonstrating the position of the L-shaped reconstruction plate on the left articular eminence. This CT scan was taken prior to performing the same procedure to the right TMJ.

\section{Case 2}

A 27-year-old female presented to our services with a chief complaint of difficulty in opening and closing her mouth with severely noted asymmetry of her mandible to the right. Her medical history included a diagnosis of myotonic dystrophy as well as Burkitt's lymphoma of her palate, which was resected and reconstructed when the patient was three years of age. A brief history revealed that the patient had undergone a full-mouth clearance of her remaining dentition under general anesthesia approximately one year prior to her initial presentation. Prior to this treatment, the patient recounted she was able to close her mouth more appropriately without any noticeable midline shift. Panoramic X-ray and CT scans were acquired and revealed that her left mandibular condyle was anteriorly dislocated from the glenoid fossa and osseous remodelling of the left condyle had occurred. Based on the clinical and radiographic findings (Figures 2-4), a diagnosis of chronic protracted left TMJ dislocation was made. After some discussion, it was decided that the best course of action was to examine the left TMJ dislocation under general anesthesia and attempt to perform a manual reduction with possible surgical intervention to obtain suitable reduction. Under general anesthesia, multiple attempts at closed reduction using considerable force were unsuccessful, and surgical exposure of the left TMJ was deemed necessary. The left TMJ was exposed using an extended pre-auricular approach with an anterosuperior (hockey-stick) extension. Direct examination of the left condyle revealed that osseous remodelling had formed a pseudo-articulation anterior to the articular eminence of the temporal bone, with overlying dense fibrous tissue. Multiple attempts at distracting the condyle from this pseudo-articulation were unsuccessful and the decision was made to perform a condylectomy to free the remaining condylar neck from the fibrous ankylosis. An osteotomy using a 701 bur was established $1 \mathrm{~cm}$ below the articulating facet of the condyle. A condylectomy was then carried out using a combination of mallet and osteotome to prevent vascular injury. A lateral pterygoid myotomy was then performed and the remaining condylar neck was smoothed using a combination of bur and rasp under copious irrigation. The mandible was then manually manipulated to visibly assess re-approximation of the ramus and existing condylar neck back into the glenoid fossa. When this was assured, the capsule was then sutured to the superior temporalis fascia to encourage a posterior seating of the condylar neck using 3-0 Vicryl sutures. Subcutaneous tissue and temporoparietal fascia were resuspended using 3-0 Vicryl buried stitches and the skin was closed using a running 4-0 Monocryl suture in a subcuticular fashion. Because of the patient's lack of dentition, maxillo-mandibular fixation (MMF) was not applied post-operatively. Over the following days, her recovery was uneventful and she was able to tolerate a soft diet without any complications or further dislocations. 
Citation: Elgazzar R, Ward C (2018) Surgical Management of Chronic and Refractory TMJ Dislocation in Medically Compromised Patients. Dentistry 8: 510. doi:10.4172/2161-1122.1000510

Page 3 of 5
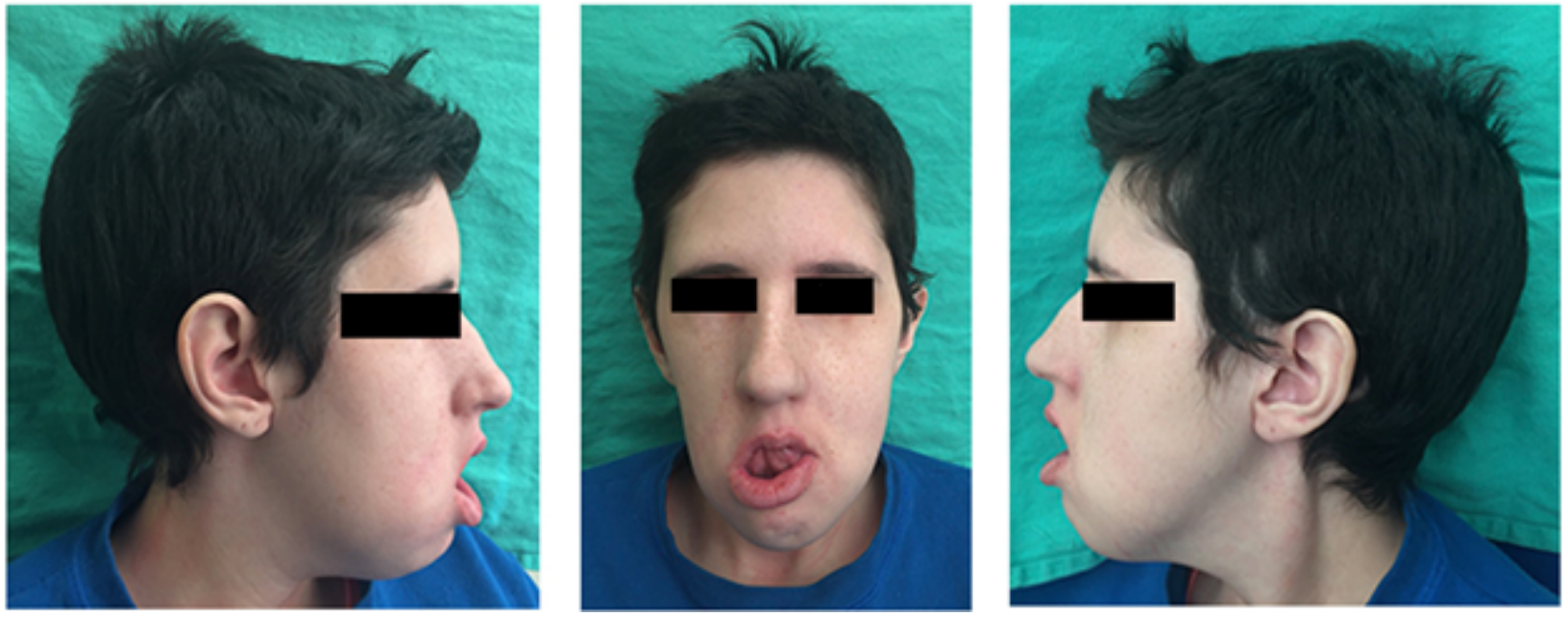

Figure 2: Pre-operative view of patient with long-standing chronic protracted dislocation.

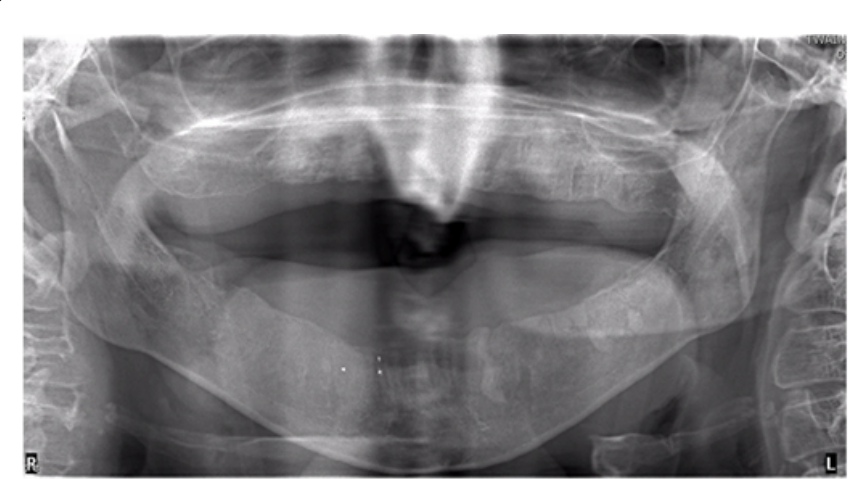

Figure 3: Pre-operative panoramic radiograph demonstrating anterior displacement of the left condyle.

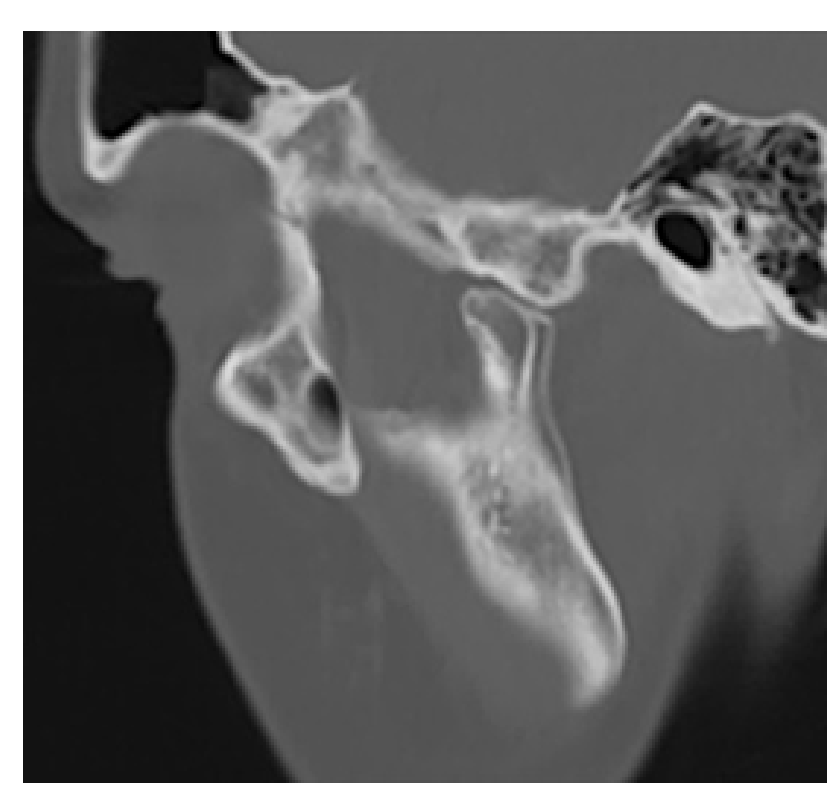

Figure 4: Sagittal view of the left chronic protracted TMJ dislocation obtained by CT imaging. Radiographic signs of osseous remodelling to the condylar head can be seen.

Improved speech, a reported improvement in masticatory function and correction of the mandibular deviation and facial asymmetry previously observed were all noted at the patient's one-week postoperative appointment (Figures 5 and 6). The patient was then placed on a regimen of TMJ physiotherapy with adjunctive TMJ home exercises to improve her range of motion. Overall, the pre-auricular incisions healed well resulting in an inconspicuous scar and the patient's recovery was free of any post-operative complications. No recurrent dislocations or degenerative joint changes have been noted in the 12 months following her surgery. 
Citation: Elgazzar R, Ward C (2018) Surgical Management of Chronic and Refractory TMJ Dislocation in Medically Compromised Patients.
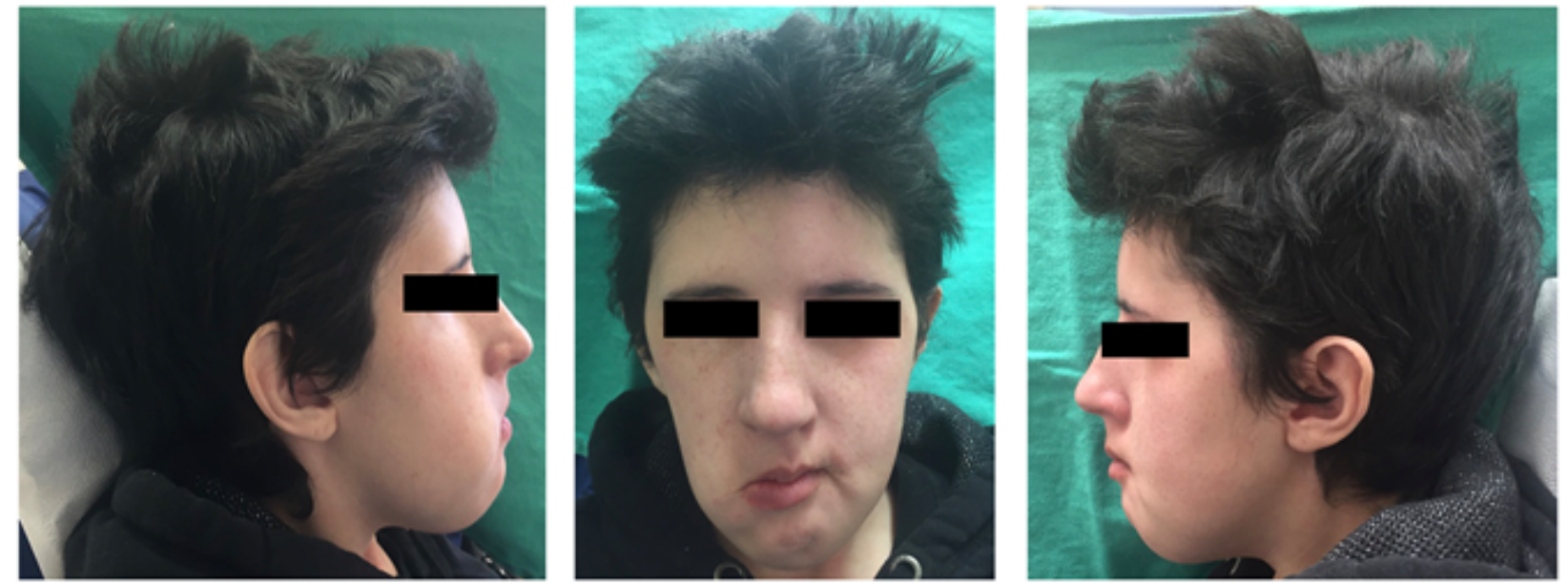

Figure 5: Post-operative view of patient with long-standing chronic protracted dislocation.

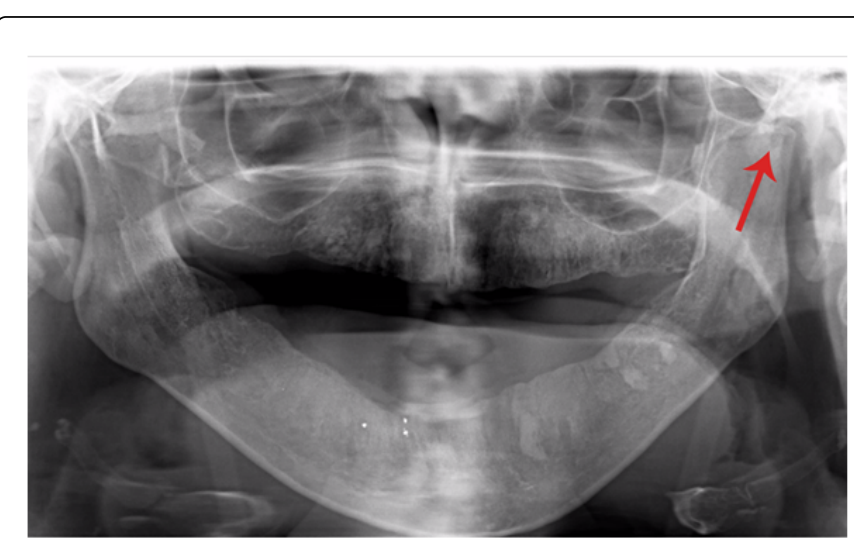

Figure 6: Post-op panoramic radiograph demonstrating left condylectomy (arrow).

\section{Discussion}

TMJ dislocation typically presents in an acute episode in which the condyles are unilaterally or bilaterally displaced anterior to the articular eminence or outside of the glenoid fossa. It is crucial that the signs and symptoms of an acute TMJ dislocation are recognised and that it is treated in a timely manner to avoid the consequences associated with chronic TMJ dislocation. In circumstances of chronic protracted TMJ dislocation, the new position of the condyle can result in fibrosis and scar formation in the joint capsule, and if not addressed, the formation of a pseudo joint. These physiological changes that occur in cases of chronic protracted TMJ dislocation make reduction challenging, and often necessitate surgical intervention. Likewise, in cases involving chronic recurrent TMJ dislocation, the frequency and duration of the dislocation episodes are important factors that can help predict the efficacy of the chosen treatment modality. Choosing an appropriate treatment modality for both chronic protracted and recurrent TMJ dislocation can be challenging due to the infrequency of these conditions, lack of definitive treatment guidelines, and a multitude of both conservative and surgical treatment options that have been described to date. With chronic recurrent TMJ dislocation, the stability of joint can be affected over time as changes to the joint capsule, ligaments, and bony architecture of joint occur. In this case report, a patient's underlying Ehlers-Danlos syndrome caused laxity in her joint capsule and ligaments, resulting in chronic recurrent dislocations when excessive force was placed on the mandible or after excessively wide mouth opening. Management of the patient's condition was approached in a stepwise fashion, employing multiple conservative treatment options until it was evident that surgical intervention was required. Manual reduction, IMF, injection of autologous blood and sclerosing agents were all employed without long-term success. Broadly, surgical intervention to correct chronic recurrent TMJ dislocation can be categorized as either augmenting or reducing the articular eminence. Proponents of utilising eminectomy procedures to alter the bony architecture of the TMJ advocate that the procedure is simpler than augmentation [6]. However, some reports suggest that eminectomies should be reserved for patients with advanced age, loss of dentition and vertical dimension, disabilities that affect voluntary muscle control, and where augmentation procedures have been unsuccessful [6]. Given the age of our patient at the time of surgery and the inherent hypermobility of her TMJs due to EhlersDanlos, augmentation of the eminences with thick L-shaped thick plates and stabilization with a temporalis myofascial flap and lateral pterygoid myotomy was chosen over performing bilateral eminectomies. In addition to stabilizing the laxity of the joint by reinforcing the capsule, the temporalis myofascial flap also provides some degree of cushioning between the condyle and the augmented eminence [6].

As with cases involving chronic recurrent TMJ dislocations, many surgical methods to reduce chronic protracted TMJ dislocations have been described in the literature. However, no distinct protocols or guidelines exist in regards to which intervention is most appropriate in a given situation. The main objective of surgical intervention is to return the condyle back to its physiologic position in the glenoid fossa and maintain this physiologic position after less invasive methods have failed to do so. Procedures such as eminectomy, condylotomy, myotomy, and menisectomy have all been employed to aid in restoring the condyle back to a normal position. However, these procedures are only successful if there has been little to no changes in the structural 
Citation: Elgazzar R, Ward C (2018) Surgical Management of Chronic and Refractory TMJ Dislocation in Medically Compromised Patients.

Page 5 of 5

integrity of the joint itself [7]. Other methods that employ the use of orthognathic surgical procedures such as bilateral sagittal split osteotomies and inverted L-osteotomies circumvent the risks associated with open joint surgery and are used to manage malocclusion, while restoring some function when adequate reduction of the joint is not possible [7]. Overall, the consensus among the reports on chronic protracted TMJ dislocation agrees that adequate reduction becomes more difficult over time. In this case, a treatment strategy like that outlined by Huang, et al. [8] was employed. These authors suggested that dislocations lasting greater than 3 weeks be treated with closed reduction under sedation or general anesthesia. Those lasting 1-3 months generally require open reduction and stripping of the muscles of mastication and associated periosteum under traction, while those greater than 6 months require open reduction in combination with condylectomy, condylotomy, myotomy or total joint reconstruction. The patient in our case could not recall when her dislocation had occurred, but records indicated her previous dental extractions occurred more than 1 year before the open joint surgery. Closed reduction with the aid of traction under general anesthesia was initially attempted without success, resulting in the decision to utilize an open joint reduction. When a pseudo joint was observed during the approach, and given that the patient was edentulous, it became evident that our treatment options would be limited to condylectomy or total joint reconstruction. Overall, the condylectomy was the only available procedure to adequately return the remaining condylar neck back to its physiologic position after other modalities had failed to do so.

\section{Conclusion}

These two cases outline the difficulties experienced when treating chronic protracted TMJ dislocation and chronic recurrent TMJ dislocation. Although, there are no definitive guidelines or protocols for treatment of these two conditions, it is crucial to approach both conditions in a stepwise manner. The age of the patient, etiology, functional status, and frequency and duration of the dislocation all should be taken into consideration if surgical intervention is necessary. Overall, both cases demonstrate that a series of interventions were performed before successful long-term stability of the TMJ was achieved. Although both cases required surgical intervention, both patients had an uneventful post-operative recovery period with no reported relapses in the 24 months following their respective surgeries.

\section{Funding}

None

\section{Disclosure}

None

\section{References}

1. Jeyaraj P, Chakranarayan A (2016) A conservative surgical approach in the management of longstanding chronic protracted temporomandibular joint dislocation: a case report and review of literature. J Maxillofac Oral Surg 15: 361-370.

2. Martins WD, Ribas Mde O, Bisinelli J, Franca BH, Martins G (2014) Recurrent dislocation of the temporomandibular joint: a literature review and two case reports treated with eminectomy. Cranio 32: 110-117.

3. Akinbami BO (2011) Evaluation of the mechanism and principles of management of temporomandibular joint dislocation. Systematic review of literature and a proposed new classification of temporomandibular joint dislocation. Head Face Med 7: 10.

4. Kim CH, Kim DH (2012) Chronic dislocation of temporomandibular joint persisting for 6 months: a case report. 38: 305-309.

5. Terakado N, Shintani S, Nakahara Y, Yano J, Hino S, et al. (2006) Conservative treatment of prolonged bilateral mandibular dislocation with the help of an intermaxillary fixation screw. Br J Oral Maxillofac Surg 44: 62-63.

6. Ying B, Hu j, Zhu S (2013) Modified Leclerc blocking procedure with miniplates and temporal fascial flap for recurrent temporomandibular joint dislocation. J Craniofac Surg 24: 740-742.

7. Marques-Mateo M, Puche-Torres M, Iglesias-Gimilio ME (2016) Temporomandibular chronic dislocation: The long-standing condition. Med Oral Patol Oral Cir Bucal 21: e776-e783.

8. Huang IY, Chen CM, Kao YH, Chen CM, Wu CW (2011) Management of long-standing mandibular dislocation. Int J Oral Maxillofac Surg 40: 810-814. 